\title{
Surface gravity and effective temperature of the K5 subdwarf G 224-58 A and Arcturus from the fits to Ti and MgH lines
}

\author{
I. O. Kushniruk ${ }^{1 *}$ Ya. V. Pavlenko ${ }^{2,3}$, M. C. Gálvez-Ortiz ${ }^{4}$, Z. H. Zhang ${ }^{5,6}$ \\ ${ }^{1}$ Taras Shevchenko National University of Kyiv, Glushkova ave., 4, 03127, Kyiv, Ukraine \\ ${ }^{2}$ Main Astronomical Observatory of the NAS of Ukraine, 27 Akademika Zabolotnoho St., 03680 Kyiv, Ukraine \\ ${ }^{3}$ Centre for Astrophysics Research, University of Hertfordshire, College Lane, Hatfield, Hertfordshire AL10 9AB, UK \\ ${ }^{4}$ Centro de Astrobiología (CSIC-INTA), Crta, Ajalvil km 4. E-28850, Torrejón de Ardoz, Madrid, Spain \\ ${ }^{5}$ Instituto de Astrofísica de Canarias, E-38205 La Laguna, Tenerife, Spain \\ ${ }^{6}$ Universidad de La Laguna, Dept. Astrofísica, E-38206 La Laguna, Tenerife, Spain
}

\begin{abstract}
We discuss here the results obtained in the determination of effective temperatures and metallicities of late-type stars. We provide the abundances of $\mathrm{MgH}$, Ti i and $\mathrm{Ti}$ II, with a list of selected lines, for the red giant of Arcturus and the metal-poor K subdwarf G 224-58 A. The Sun was used as a template star. After computing synthetic spectra for model atmospheres with different $T_{\text {eff }}$ and $\log g$ values, we used minimization procedures to determine the best fits to the observed features. The determination of Ti abundances was carried out in the framework of a self-consistent approach developed by Pavlenko et al. (2012). In the case of $\mathrm{MgH}$ we used similar minimization procedure. We present here the combination of two different approaches based on the fits performed to $\mathrm{Ti}$ and $\mathrm{MgH}$, respectively. The resulting effective temperatures are $T_{\text {eff }}=4300 \mathrm{~K}$ for Arcturus and $T_{\text {eff }}=4600 \mathrm{~K}$ for $\mathrm{G} 224-58 \mathrm{~A}$, where both solutions are degenerated with $\log g$.
\end{abstract}

Key words: stars: atmospheres, stars: fundamental parameters, stars: individual (Arcturus), stars: individual (G 224-58A), line: profiles

\section{INTRODUCTION}

Any analysis of spectra of late-type stars begins with the determination of their main physical parameters, i.e. effective temperature $\left(T_{\text {eff }}\right)$ and gravity $(\log g)$.

In most of the cases it can be done using fits to $\mathrm{Fe}, \mathrm{Ti}$ and molecular $\mathrm{MgH}$ lines in observed spectra. From ionization balance $(\log [\mathrm{XI} / \mathrm{H}] \simeq \log [\mathrm{XII} / \mathrm{H}])$ we obtain a group of solutions for $T_{\text {eff }}$ and $\log g$. In the case of late-type stars, appropriate lines of Fe I and Ti, Fe II and Ti II ions are available. Balmer lines show rather marginal sensitivity on $\log g$, therefore we use other line analysis to determine $\log g$.

Gravities have been measured based on Fe lines for a sample of late-type stars in $[6,17]$. But Ti I and Ti II line analysis is preferable at lower $T_{\text {eff }}$ due to lower ionization potential of $\mathrm{Ti} \chi=6.8281 \mathrm{eV}$ vs. $\chi=7.9024 \mathrm{eV}$ for Fe I [5]. MgH molecular lines were also used to obtain $\log g$ in [7]. In our paper [4] $\mathrm{MgH}$ line and an updated value of its dissociation potential were used to obtain $\log g$ and $T_{\text {eff }}$ for Arcturus.

G 224-58 AB is a wide binary ( 93 arcsec in separation) of spectral type esdK5+esdM5.5. It was identified by motion, colour and spectroscopy in [18]. $\mathrm{G} 224-58 \mathrm{~B}$ is an M extreme metal deficient subdwarf.
These M subdwarfs are the most numerous stars in the Milky Way. They can be used to verify theories on Galaxy evolution and exoplanet searches. By modelling the spectra of the primary, the authors of [12] determined chemical abundances, and $T_{\text {eff }} \log g /[\mathrm{Fe} / \mathrm{H}]=4625 / 4.5 /-2.0$ for the $\mathrm{G} 224$ $58 \mathrm{~A}$.

In this paper we investigate the problem of determination of $\log g$ and $T_{\text {eff }}$ for Arcturus and G 224$58 \mathrm{~A}$ using the fine and self-consistent analysis of Ti I, Ti II and MgH lines.

\section{OBSERVATIONS}

\section{ARCTURUS AND THE SUN}

We usually take the Sun and Arcturus as template stars. Their high-resolution spectra are wellstudied by many authors, such as [13] and references therein. We took the observed spectrum of Arcturus from [1] and solar spectrum from [3].

High-resolution spectrum of G 224-58 A was observed on 2013 June 19th with FIES [16] at the Nordic Optical Telescope. The signal-to-noise ratio of the spectrum is $\sim 35$ in $\mathrm{H} \alpha$ line. For more details on observations and reduction procedures please see [12].

\footnotetext{
*nondanone@gmail.com

(C) I. O. Kushniruk, Ya. V.Pavlenko, M. C. Gálvez-Ortiz, Z. H. Zhang, 2016
} 

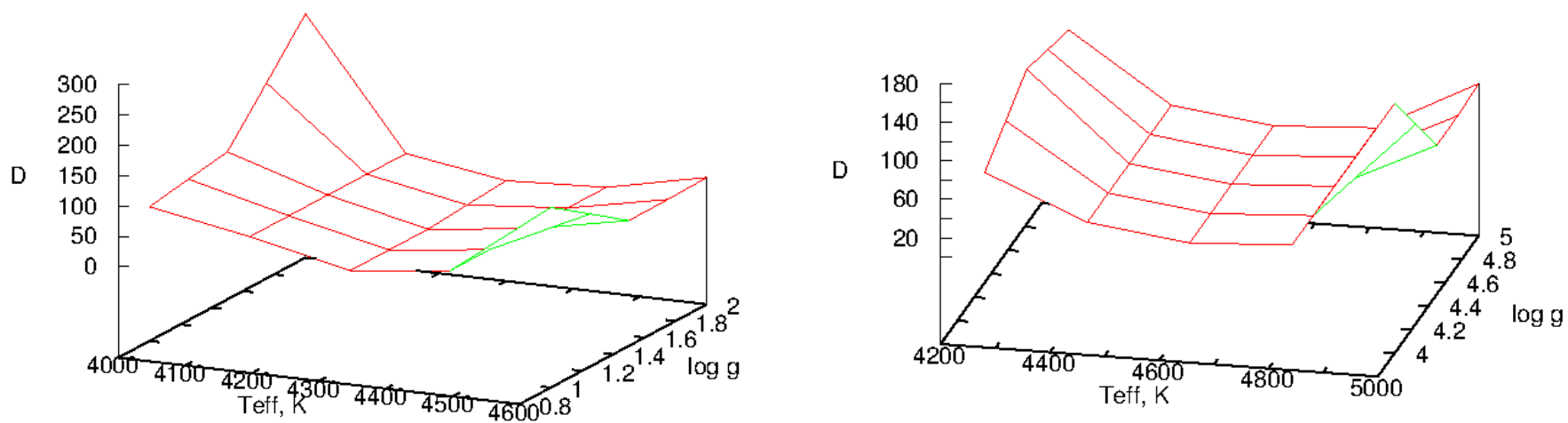

Fig. 1: $\log g$ and $T_{\text {eff }}$ dependence of D-function values for Arcturus (left) and G 224-58 A (right).

\section{THE PROCEDURE}

\section{MODEL ATMOSPHERES, SYNTHETIC SPECTRA}

We carried out our analysis in the framework of classical approach: local thermal equilibrium (LTE), 1D model atmospheres, no sources or sinks. On the first step of our work we used the plane-parallel model atmosphere $5777 / 4.44 / 0.0$ described in [10] for the Sun, the PDK (Peterson-Dalle-Kurucz) [13] model atmosphere with parameters $4300 / 1.5 /-0.3$ for Arcturus, and the 4625/4.5/ - 2.0 model atmosphere from our recent paper [12] for G 224-58 A.

Synthetic spectra were computed by WITA6 code [8]. We used atomic and $\mathrm{MgH}$ list from the VALD3 database [15] and Kurucz database [2], respectively. To determine abundances of $\mathrm{Ti}$ and $\mathrm{Mg}$ we used the ABEL8code [9] (see more details in [11]).

We computed small grids of model atmospheres by SAM12 code [10], with different effective temperatures and gravities for each of the problem stars. In general, we computed 21 models for each star with $100 \mathrm{~K}$ step in $T_{\text {eff }}$ and 0.5 step in $\log g$. See Table 1 for more details in model atmosphere parameters.

\section{MICROTURBULENT VELOCITY}

We adopted $V_{t}=0.75 \mathrm{~km} / \mathrm{s}$ for the Sun based on 48 specially selected Fe I lines and $V_{t}=1 \mathrm{~km} / \mathrm{s}$ for Arcturus based on $44 \mathrm{Fe}$ I lines, according to [12]. We adopted $V_{t}=0.5 \mathrm{~km} / \mathrm{s}$ for the $\mathrm{G} 224-58 \mathrm{~A}$ atmosphere (see $[11,12])$.

\section{TI I AND TI II LINES}

Selection of Ti line list is an important step in the abundance determination procedure. We used the full atomic VALD3 list [15] to compute the synthetic spectra of the Sun in the optical range. Then we excluded Ti I and Ti II lines from the input line list for WITA6 code [8] and re-computed spectra. From the comparison of these two theoretical spectra of the Sun we obtained Ti I and Ti II lines. Then every line in the observed spectrum was identified. We changed abundance of $\mathrm{Ti}$ on \pm 0.2 dex and chose the most suitable lines. Lines affected by severe blending in the observed spectrum were excluded. Abundances of Ti were computed separately for the preselected Ti I and Ti II lines lists. For every model atmosphere we computed $\Delta \log N(x)=|\log \mathrm{TiI}-\log \mathrm{TiII}| \simeq 0$ (see Table 1).

\section{$\mathrm{MGH}$}

The strength of $\mathrm{MgH}$ lines in the theoretical spectra is sensitive to both $\log g$ and $T_{\text {eff }}$. Thus, modelling $\mathrm{MgH}$ lines can be used to determine these stellar parameters.

Similarly to Ti case, we divided synthetic spectra "MgH + atomic lines" by "atomic lines", and obtained pure $\mathrm{MgH}$ spectrum. Then we selected the better lines present in observed spectra of our objects. The next step was computing synthetic spectra for our model atmospheres, and after that parameter $S$ was obtained from equation:

$$
S=\sum_{i=1}^{n} \frac{\left(f_{\text {observed }_{i}}-f_{\text {computed }_{i}}\right)^{2}}{\lambda_{2}-\lambda_{1}}, \quad S_{\mathrm{MgH}}=\sum_{j=1}^{N} S_{j}
$$

where $n$ is the number of points in the feature, $N$ is the number of features, $S_{j}$ is the deviation for each feature, $S$ is the total deviation for the model atmosphere with some $\log g$ and $T_{\text {eff }}$ from the grid, $\lambda_{1}$ and $\lambda_{2}$ are the limiting wavelengths of $\mathrm{MgH}$ feature.

We used $S_{\mathrm{MgH}}$ to estimate the quality of fits of our synthetic spectra to the observed spectra. In Table 1 we show $S_{\mathrm{MgH}}$ obtained by fits to $\mathrm{MgH}$ lines from observed spectra of Arcturus and G 224-58 A. MgH lines are too weak in the solar spectrum to carry out their analysis for the Sun.

Finally, for Arcturus and G 224-58 A we computed the "combined function":

$$
D=S_{\mathrm{MgH}} \times 10^{\mid \log N(\text { TiI })-\log N(\text { TiII }) \mid}
$$

and investigated its dependence on $\log g$ and $T_{\text {eff }}$. 


\section{RESULTS AND DISCUSSION}

We have computed abundances of Titanium using fits to $\mathrm{Ti}$ I and $\mathrm{Ti}$ II lines and provided fits to the observed $\mathrm{MgH}$ lines in the spectra of Arcturus and G 224-58 A for all our model atmospheres. Results are shown in Table 1 and Fig. 1.

Arcturus is one of the most studied stars after the Sun, and many authors have previously determined its fundamental parameters from its high-resolution spectra. A short overview can be found in [4]. One of the most recent and precise results is found in [14], that give $T_{\text {eff }}=4286 \pm 30 \mathrm{~K}$ and $\log g=1.66 \pm 0.05$. As can be seen in Table 1 and Fig. 1, we derived a $T_{\text {eff }}=4300 \mathrm{~K}$ for Arcturus, what is in good agreement with [14].

For both Arcturus and G 224-58 A, we have found that our $\mathrm{D}$ function is dependent on $T_{\text {eff }}$ and $\log g$. Therefore, once we determined $T_{\text {eff }}$, we fixed it and determined $\log g$.

For Arcturus, fixing of $T_{\text {eff }}=4300 \mathrm{~K}$, the smallest value of deviation in $\mathrm{D}$ according to Ti lines, gives the value of $\log g=2.0$. According to $\mathrm{MgH}$ results, $\log g=1.0$. We consider that the best value is probably an average of both, $\log g=1.5$.

For G 224-58 A, when we fix $T_{\text {eff }}$ to $4600 \mathrm{~K}$ (the best value, see Fig. 1; see detailed analysis in [12]), Ti lines methodology provides a value of $\log g=4.5$, while $\mathrm{MgH}$ method gives $\log g=4.0$. Again, we have a degeneration of surface gravity. Values obtained here are very close to the ones obtained in [12], $T_{\text {eff }}=4625 \pm 100 \mathrm{~K}$ and $\log g=4.5 \pm 0.5$.

Despite the low number of the fitted lines in the solar spectrum we found the local minima of $\Delta \log N(x)$ around values 5750/5.0.

We conclude that the combination of fitting $\mathrm{MgH}$ and Ti lines in high-resolution spectra does not cancel the degeneracy of solutions in respect to the surface gravity. Some additional constraints should be used to determine both $T_{\text {eff }}$ and $\log g$.

\section{ACKNOWLEDGEMENT}

We thank Profs. Hinkle, Kurucz, and VALD3 team for providing databases of astrophysical data used in our work and anonymous Referee for some positive remarks.

\section{REFERENCES}

[1] Hinkle K., Wallace L., Valenti J. \& Harmer D. 2000, 'Visible and Near Infrared Atlas of the Arcturus Spectrum 3727-9300 A', ASP, San Francisco

[2] Kurucz R. \& Bell B. 1995, Atomic Line Data Kurucz CD-ROM No. 23, Cambridge, Mass.: Smithsonian Astrophysical Observatory

[3] Kurucz R. L., Furenlid I., Brault J. \& Testerman L. 1984, in 'National Solar Observatory Atlas', Sunspot, New Mexico

[4] Kushniruk I. O., Pavlenko Ya. V. \& Kaminskiy B. M. 2013, Advances in Astronomy and Space Physics, 3, 29

[5] LideD. 2003, 'CRC Handbook of Chemistry and Physics', CRC Press. Boca Raton, Florida

[6] Lind K., Bergemann M. \& Asplund M. 2012, MNRAS, 427,50

[7] Lyubimkov L. 1995, 'Chemical composition of stars: method and result of analysis', Astroprint, Odessa

[8] Pavlenko Ya. V. 1997, Ap\&SS, 253, 43

[9] Pavlenko Ya. V. 2002, Kinematika i Fizika Nebesnykh Tel, 18, 1, 48

[10] Pavlenko Ya. V. 2003, Astron. Rep., 47, 59

[11] Pavlenko Ya. V., Jenkins J. S., Jones H. R. A., Ivanyuk O. \& Pinfield D. J. 2012, MNRAS, 422, 542

[12] Pavlenko Ya. V., Zhang Z. H., Gálvez-Ortiz M. C., Kushniruk I. O. \& Jones H. R. A. 2015, A\&A, 582, A92

[13] Peterson R. C., Dalle Ore C. M. \& Kurucz R. L. 1993, ApJ, 404, 333

[14] Ramírez I. \& Allende Prieto C. 2011, ApJ, 743, 135

[15] Ryabchikova T. A., Pakhomov Yu. V. \& Piskunov N. E. 2011, Uchenye zapiski Kazanskogo Universiteta, 153, 61

[16] Telting J. H., Avila G., Buchhave L. et. al. 2014, Astronomische Nachrichten, 335, 1, 41

[17] Thévenin F. \& Idiart T. P. 1999, ApJ, 521, 753

[18] Zhang Z.H., Pinfield D. J., Burningham B. et al. 2013, MNRAS, 434, 1005 
Table 1: Changes of parameters of deviation found in the variations of $T_{\text {eff }}$ and $\log g$ for $\mathrm{Ti}$ and $\mathrm{MgH}$ lines.

\begin{tabular}{|c|c|c|c|c|c|c|c|c|c|c|}
\hline \multicolumn{4}{|c|}{ Arcturus } & \multicolumn{4}{|c|}{ G 224-58 A } & \multicolumn{3}{|c|}{ Sun } \\
\hline$T_{\text {eff }}$ & $\log g$ & $\Delta \log N(\mathrm{x})$ & $S_{\mathrm{MgH}}$ & $T_{\text {eff }}$ & $\log g$ & $\Delta \log N(\mathrm{x})$ & $S_{\mathrm{MgH}}$ & $T_{\text {eff }}$ & $\log g$ & $\Delta \log N(\mathrm{x})$ \\
\hline 4000 & 1.0 & $0.21 \pm 0.18$ & $43.68 \pm 0.79$ & 4250 & 4.0 & $0.287 \pm 0.053$ & $33.90 \pm 0.83$ & 5500 & 4.0 & $0.25 \pm 0.07$ \\
\hline 4000 & 1.5 & $0.18 \pm 0.13$ & $62.07 \pm 1.04$ & 4250 & 4.5 & $0.502 \pm 0.053$ & $35.21 \pm 0.83$ & 5500 & 4.5 & $0.25 \pm 0.05$ \\
\hline 4000 & 2.0 & $0.49 \pm 0.05$ & $81.67 \pm 1.28$ & 4250 & 5.0 & $0.425 \pm 0.044$ & $34.48 \pm 0.83$ & 5500 & 5.0 & $0.45 \pm 0.04$ \\
\hline 4100 & 1.0 & $0.06 \pm 0.20$ & $22.90 \pm 0.46$ & 4500 & 4.0 & $0.064 \pm 0.065$ & $7.73 \pm 0.39$ & 5650 & 3.5 & $0.25 \pm 0.06$ \\
\hline 4100 & 1.5 & $0.01 \pm 0.10$ & $40.65 \pm 1.33$ & 4500 & 4.5 & $0.175 \pm 0.044$ & $13.21 \pm 0.57$ & 5650 & 4.0 & $0.31 \pm 0.07$ \\
\hline 4100 & 2.0 & $0.28 \pm 0.09$ & $57.30 \pm 0.98$ & 4500 & 5.0 & $0.334 \pm 0.041$ & $16.69 \pm 0.67$ & 5650 & 4.5 & $0.18 \pm 0.05$ \\
\hline 4200 & 1.0 & $0.16 \pm 0.21$ & $4.59 \pm 0.14$ & 4600 & 4.0 & $0.173 \pm 0.060$ & $3.39 \pm 0.12$ & 5650 & 5.0 & $0.36 \pm 0.05$ \\
\hline 4200 & 1.5 & $0.16 \pm 0.11$ & $17.00 \pm 0.38$ & 4600 & 4.5 & $0.025 \pm 0.044$ & $6.51 \pm 0.34$ & 5750 & 3.5 & $0.10 \pm 0.12$ \\
\hline 4200 & 2.0 & $0.05 \pm 0.05$ & $34.52 \pm 0.66$ & 4600 & 5.0 & $0.218 \pm 0.059$ & $9.97 \pm 0.52$ & 5750 & 4.0 & $0.08 \pm 0.12$ \\
\hline 4300 & 1.0 & $0.30 \pm 0.21$ & $2.29 \pm 0.08$ & 4650 & 4.0 & $0.291 \pm 0.062$ & $5.47 \pm 0.26$ & 5750 & 4.5 & $0.40 \pm 0.10$ \\
\hline 4300 & 1.5 & $0.07 \pm 0.18$ & $3.573 \pm 0.11$ & 4650 & 4.5 & $0.075 \pm 0.044$ & $3.76 \pm 0.16$ & 5750 & 5.0 & $0.01 \pm 0.02$ \\
\hline 4300 & 2.0 & $0.01 \pm 0.12$ & $15.20 \pm 0.35$ & 4650 & 5.0 & $0.007 \pm 0.046$ & $6.08 \pm 0.36$ & 5777 & 4.4 & $0.20 \pm 0.01$ \\
\hline 4400 & 1.0 & $0.28 \pm 0.27$ & $13.52 \pm 0.32$ & 4750 & 4.0 & $0.425 \pm 0.044$ & $11.31 \pm 0.34$ & 5900 & 3.5 & $0.48 \pm 0.01$ \\
\hline 4400 & 1.5 & $0.09 \pm 0.22$ & $2.38 \pm 0.08$ & 4750 & 4.5 & $0.175 \pm 0.044$ & $4.78 \pm 0.12$ & 5900 & 4.0 & $0.25 \pm 0.12$ \\
\hline 4400 & 2.0 & $0.05 \pm 0.15$ & $3.20 \pm 0.11$ & 4750 & 5.0 & $0.007 \pm 0.037$ & $4.19 \pm 0.12$ & 5900 & 4.5 & $0.11 \pm 0.12$ \\
\hline 4500 & 1.0 & $0.43 \pm 0.19$ & $31.62 \pm 0.81$ & 5000 & 4.0 & $0.625 \pm 0.025$ & $40.06 \pm 0.93$ & 5900 & 5.0 & $0.13 \pm 0.02$ \\
\hline 4500 & 1.5 & $0.21 \pm 0.14$ & $13.09 \pm 0.30$ & 5000 & 4.5 & $0.375 \pm 0.025$ & $27.21 \pm 0.65$ & 6000 & 3.5 & $0.14 \pm 0.02$ \\
\hline 4500 & 2.0 & $0.15 \pm 0.05$ & $2.36 \pm 0.08$ & & & & & 6000 & 4.0 & $0.31 \pm 0.05$ \\
\hline 4600 & 1.0 & $0.48 \pm 0.10$ & $49.24 \pm 1.36$ & & & & & 6000 & 4.5 & $0.00 \pm 0.13$ \\
\hline 4600 & 1.5 & $0.28 \pm 0.10$ & $30.12 \pm 0.74$ & & & & & 6000 & 5.0 & $0.08 \pm 0.03$ \\
\hline
\end{tabular}

